\title{
Impacto de ácidos graxos essenciais na terapêutica suplementar da dismenorreia primária: uma revisão de ensaios clínicos
}

Impact of fatty acids essences on the supplementary therapeutic of primary dysmenorrhea: a review of clinical trials

Impacto de los ácidos grasos esenciales en la terapia complementaria de la dismenorrea primaria: una revisión de ensayos clínicos

Recebido: 13/10/2021 | Revisado: 19/10/2021 | Aceito: 27/10/2021 | Publicado: 30/10/2021

\author{
Maiara Moeme Alvim Guimarães \\ ORCID: https://orcid.org/0000-0002-6290-967X \\ Universidade do Estado da Bahia, Brasil \\ E-mail: maiaramoeme.nut@gmail.com \\ Edilene Maria Queiroz Araújo \\ ORCID: https://orcid.org/0000-0002-3900-5525 \\ Universidade do Estado da Bahia, Brasil \\ E-mail: dilaq@msn.com \\ Aníbal de Freitas Santos Junior \\ ORCID: https://orcid.org/0000-0002-3022-0771 \\ Universidade do Estado da Bahia, Brasil \\ E-mail: afjunior@uneb.br \\ Ana Gabriela Álvares Travassos \\ ORCID: https://orcid.org/0000-0001-9242-828X \\ Universidade do Estado da Bahia, Brasil \\ E-mail: atravassos@uneb.br
}

\section{Resumo}

Dismenorreia primária (DP) é a denominada cólica menstrual e o aparecimento de sintomas pode estar associado à ação das prostaglandinas (PG) e leucotrienos (LT). Os ácidos graxos essenciais (AGE), principalmente o ômega 3, possuem propriedades anti-inflamatórias inibitórias da síntese de PG. Assim, esta revisão sistemática tem como objetivo discutir o uso da suplementação de AGE no tratamento de DP. Estudos originais do tipo ensaio clínico, publicados entre 2000 a 2021 foram pesquisados nos bancos de dados Pubmed e Scielo. Oito artigos foram incluídos e analisados neste estudo. A partir dos resultados afirma-se que os AGE, sobretudo ômega 3, são relevantes no tratamento da DP, identificando-se como mecanismo de ação a inibição da atividade da ciclo-oxigenase pelo ômega-3, que leva a menor produção de PG e LT pró-inflamatórias, aumentando a formação de seus derivados, antiinflamatórios. Logo, sugere-se que a suplementação dietética a partir de $300 \mathrm{mg}$ de ômega-3, 8-2 dias antes e até 3-2 dias após a menstruação, principalmente após 2 meses, reduz dor em mulheres com DP.

Palavras-chave: Dismenorreia primária; Ácidos graxos essenciais; Ômegas.

\begin{abstract}
Primary dysmenorrhea (PD) is called menstrual cramps and the appearance of symptoms may be associated with the action of prostaglandins (PG) and leukotrienes (LT). Essential fatty acids (AGEs), mainly ômega 3, have antiinflammatory properties that inhibit PG synthesis. Thus, this systematic review aims to discuss the use of AGE supplementation in the treatment of PD. Original clinical trial studies, published between 2000 and 2021 , were searched in the Pubmed and Scielo databases. Eight articles were included and analyzed in this study. Based on the results, it is stated that AGEs, especially omega 3, are relevant in the treatment of PD, identifying as a mechanism of action the inhibition of cyclooxygenase activity by ômega-3, which leads to lower production of PG and LT proinflammatory, increasing the formation of its derivatives, anti-inflammatory. Therefore, it is suggested that dietary supplementation from $300 \mathrm{mg}$ of ômega-3, 8-2 days before and up to 3-2 days after menstruation, especially after 2 months, reduces pain in women with PD.
\end{abstract}

Keywords: Primary dysmenorrhea; Essential fatty acids; Omegas. 


\begin{abstract}
Resumen
La dismenorrea primaria (DP) se denomina calambres menstruales y la aparición de síntomas puede estar asociada a la acción de las prostaglandinas (PG) y los leucotrienos (LT). Los ácidos grasos esenciales (AGE), principalmente omega 3, tienen propiedades antiinflamatorias que inhiben la síntesis de PG. Por lo tanto, esta revisión sistemática tiene como objetivo discutir el uso de suplementos de AGE en el tratamiento de la DP. Los estudios de ensayos clínicos originales, publicados entre 2000 y 2021, se buscaron en las bases de datos Pubmed y Scielo. En este estudio se incluyeron y analizaron ocho artículos. Con base en los resultados, se afirma que los AGE, especialmente los omega 3, son relevantes en el tratamiento de la DP, identificando como mecanismo de acción la inhibición de la actividad ciclooxigenasa por parte de los omega-3, lo que conduce a una menor producción de PG y de LT. inflamatorio, aumentando la formación de sus derivados, antiinflamatorio. Por lo tanto, se sugiere que la suplementación dietética de $300 \mathrm{mg}$ de omega-3, 8-2 días antes y hasta 3-2 días después de la menstruación, especialmente después de 2 meses, reduce el dolor en mujeres con DP.
\end{abstract}

Palabras clave: Dismenorrea primaria; Ácidos grasos esenciales; Omegas.

\title{
1. Introdução
}

O termo "dismenorreia primária" (DP) é usado para denominar cólicas menstruais, uma das queixas ginecológicas com alta prevalência e recorrência em mulheres (Alves, Yamagishi, da Silva, Júnior \& Lima, 2016). As dores aparecem, normalmente, no dia anterior ou, no primeiro dia de fluxo menstrual, desaparecendo no fim da menstruação e, geralmente, atingem o pico após 24 horas e geralmente diminuem após dois dias. A DP apresenta outros sinais e sintomas como náuseas, vômitos, constipação ou diarreia, fadiga e dores no baixo ventre, que podem irradiar para as coxas, parte inferior e superior da coluna vertebral, causar nervosismo, tonturas, cefaléia, adinamia, além de aumentar a freqüência urinária (de Araújo, da Silva, Bastos \& Ventura, 2012) (Dall'Acqua \& Bendlin, 2015). As mulheres com DP acentuada, especialmente nos primeiros dias do ciclo menstrual, podem apresentar perda de produtividade em atividades habituais e elevados índices de absenteísmo escolar, laboral e restrições de lazer (Sezeremeta et al., 2015).

Uma explicação para fisiopatologia da DP é dada pela diminuição dos níveis de progesterona no fim do ciclo ovulatório, antes da menstruação, a qual determina um aumento anormal da contratilidade uterina, reduzindo o fluxo sanguíneo ao útero (vasoconstricção) e sensibilizando os receptores musculares miometrais, causando cólicas (dor) (Dall'Acqua \& Bendlin, 2015) (Dardes, Moraes \& Santos, 2011) (Mayo, 1997). Isso ocorre porque a redução dos níveis de progesterona causa a liberação de fosfolipídios da membrana celular (ácidos graxos, ômega-6) que, sob ação da enzima fosfolipase A2, é convertida em ácido araquidônico (AA). O AA, por sua vez, será transformado em prostaglandinas (PG) e leucotrienos (LT) que irão causar a contração e vasoconstricção uterina (Dardes, Moraes \& Santos, 2011).

A grande maioria dos sintomas observados em mulheres com DP pode ser explicada pela ação da prostaglandina, em particular a PGF2- $\alpha$, presente no fluido menstrual e no endométrio, ocasionando melhoras significativas nos sintomas de acordo com a redução nos níveis de PG (Dall'Acqua \& Bendlin, 2015) (Dardes, Moraes \& Santos, 2011) (Giraldo, Eleutério Júnior \& Linhares, 2008). Logo, a severidade da dor está relacionada à maior quantidade de PG, principalmente nos primeiros dois dias da menstruação, já aumento de LT determina os sintomas sistêmicos da DP (Giraldo, Eleutério Júnior \& Linhares, 2008).

Para aliviar a dor e tratar outros sintomas, inibe-se a síntese de prostaglandinas no endométrio. Convencionalmente, utilizam-se antiinflamatórios não-esteróides (AINE), antiespasmódicos, analgésicos e contraceptivos, orais e injetáveis. Mas, têm sido utilizadas práticas alternativas como a termoterapia, crioterapia, pilates, acupuntura e outros que apresentam melhoras na sintomatologia de cólicas menstruais (Gerzson, Padilha, Braz, \& Gasparetto, 2014).

Além dessas práticas, vários suplementos nutricionais são fontes alternativas, sendo relatados como eficazes para o tratamento da DP. Dentre estes, destacam-se os ácidos graxos essenciais (AGE) - ômega 3 (EPA, DHA), ômega 6 (GLA) -, 
magnésio, zinco, vitamina B1 (tiamina) e vitamina E, entre outros (Dardes, Moraes \& Santos, 2011). Os AGE, principalmente o ômega 3, são investigados como terapêutica suplementar por possuírem propriedades anti-inflamatórias que inibem a síntese de prostaglandinas (Hudson, 2007). Diante disso, busca-se investigar como a suplementação de AGE interfere na redução de sintomas da DP. Logo, o objetivo desta revisão foi discutir o uso da suplementação de ácidos graxos essenciais e sua relevância no tratamento da DP, identificando o mecanismo de ação de AGE no tratamento, pontuando a viabilidade, segurança e efeito do uso e, possivelmente, sugerindo a dose de segurança para a utilização do suplemento.

\section{Metodologia}

Trata-se de uma revisão sistemática baseada na literatura científica, a partir de artigos científicos (Estrela, 2018; Koche, 2011; Ludke; Andre, 2013; Pereira et al., 2018). A pesquisa dos artigos foi realizada nas bases de dados Pubmed e Scielo em 2021, utilizando-se o termo de busca bibliográfica "primary dysmenorrhea", relacionando aos termos "supplementation", "supplement", "essential fatty acid", "ômega", "fish oil", "krill oil", "borage oil", "primrose oil". Na procura das publicações, foi utilizado o operador lógico AND para realizar as seguintes combinações dos termos: I-primary dysmenorrhea AND supplementation; II-primary dysmenorrhea AND supplement; III-primary dysmenorrhea AND essential fatty acid; IV-primary dysmenorrhea AND ômega; V-primary dysmenorrhea AND fish oil; VI-primary dysmenorrhea AND krill oil; VII-primary dysmenorrhea AND borage oil; VIII-primary dysmenorrhea AND primrose oil.

Foram pesquisadas também as referências bibliográficas dos artigos incluídos. Realizou-se uma primeira avaliação, tendo por base os títulos e o resumo dos artigos, rejeitando aqueles que não preencheram os critérios de inclusão ou apresentaram algum dos critérios de não inclusão. Três autores realizaram a busca e avaliação dos artigos de maneira independente, utilizando os critérios de elegibilidade predefinidos.

Foram incluídos nesta revisão, estudos originais do tipo ensaio clínico (aleatorizados ou não / controlados ou não) e, ainda, que apresentassem resultados referentes ao efeito do uso de ácidos graxos essenciais no tratamento da dismenorreia primária. Os demais critérios de inclusão foram: pacientes em qualquer faixa etária, data da publicação do estudo de 2000 a 2021, artigos publicados nos idiomas inglês e português. Não foram incluídos os estudos observacionais, aqueles realizados em animais, artigos de revisão e que relacionavam outros nutrientes isolados que não fossem os AGE no tratamento da DP.

\section{Resultados e Discussão}

Foram encontrados 54 artigos não duplicados. Após aplicar os critérios de não inclusão foram selecionados 6 artigos, a partir da leitura de títulos e resumos. Em seguida, foram analisadas as referências bibliográficas destes, identificou-se mais 2 artigos que atendiam aos critérios de elegibilidade. Por fim, o total de 8 artigos foram incluídos nesta revisão (Figura 1).

Após análise dos oito artigos completos (Tabela 1), verificou-se que cinco não utilizaram placebo (Sampalis, 2003) (Zafari, Tofighi, Aghamohammady, Behmanesh \& Rakhshaee, 2011a) (Zafari, Behmanesh \& Mohammadi, 2011b) (Rahbar, Asgharzadeh \& Ghorbani, 2012) (Mehrpooya, Eshraghi, Rabie, Larki-Harchegani \& Ataei, 2017) e três foram placebocontrolados (dois cruzados) (Moghadamnia, Mirhosseini, Abadi, Omranirad \& Omidvar, 2010) (Hosseinlou, Alinejad, Alinejad \& Aghakhani, 2014) (Sadaghi et al., 2018). Entre eles, um artigo analisa a eficácia do ômega 3 isoladamente (Moghadamnia et al., 2010), três estudos compararam a eficácia do ômega 3 a outros nutrientes (vitamina B1, cálcio e vitamina E (Hosseinlou, et al., 2014) (Mehrpooya et al., 2017) (Sadaghi et al., 2018), outro ensaio clínico comparou o ômega 3 sob as formas de óleo de peixe e krill (Sampalis, 2003) e três pesquisas avaliaram a eficácia do ômega 3 em relação ao uso do ibuprofeno (Zafari et al., 2011a) (Zafari et al., 2011b) (Mehrpooya et al., 2017), porém um destes fez comparação 
concomitantemente com acupuntura ibuprofeno (Zafari et al., 2011a). Nenhum ensaio clínico foi encontrado sobre a eficácia de ômega 6 GLA, com o uso do óleo de prímula e/ou borragem, no tratamento da DP.

O menor tempo de uso da suplementação do ômega 3 nos estudos foi de dois meses (Sampalis, 2003) (Zafari et al., 2011a) (Hosseinlou, et al., 2014) (Sadaghi et al., 2018). As dosagens descritas nos ensaios de ômega 3 utilizadas foram de 300mg (Sampalis, 2003) (Zafari et al., 2011a) (Rahbar et al., 2012) (Sadaghi et al., 2018), 755mg (Moghadamnia et al., 2010) e 1000mg (Mehrpooya et al., 2017) e dois estudos (Zafari et al., 2011b) (Sadaghi et al., 2018) não determinavam dosagens especificas de ômega 3, contido no óleo de peixe. O menor tamanho amostral foi de 36 (Moghadamnia et al., 2010), e o máximo, de 240 (Hosseinlou, et al., 2014) mulheres.

Os resultados dos ensaios clínicos selecionados mostraram que o ômega 3, de forma unânime, foi capaz de reduzir o sintoma de dor em mulher com DP, a qualquer dose utilizada. Alguns estudos que avaliaram comparativamente a redução de sintomas a cada ciclo, averiguou-se que, a partir de um mês de uso é notório redução da dor (Sampalis, 2003) (Mehrpooya et al., 2017) e todos estudos observaram que com dois meses, além da dor, há redução de outros sintomas relacionados (náusea, vômito, dor lombar e abdominal, sensibilidade da mama, acne, dores de cabeça, edema e inchaço), indicando a relevância do uso da suplementação de AGE no tratamento de DP.

Entre os artigos que comparam a suplementação de ômega 3 ao ibuprofeno, os estudos de Zafari et al. (2011a, 2011b) e Mehrpooya et al. (2017) recomendaram que o uso de ômega-3 poderia oferecer uma alternativa aos medicamentos que possuem efeitos colaterais como anti-inflamatórios não esteróides (AINES), para um tratamento seguro e eficaz. 
Figura 1 - Fluxograma da busca.

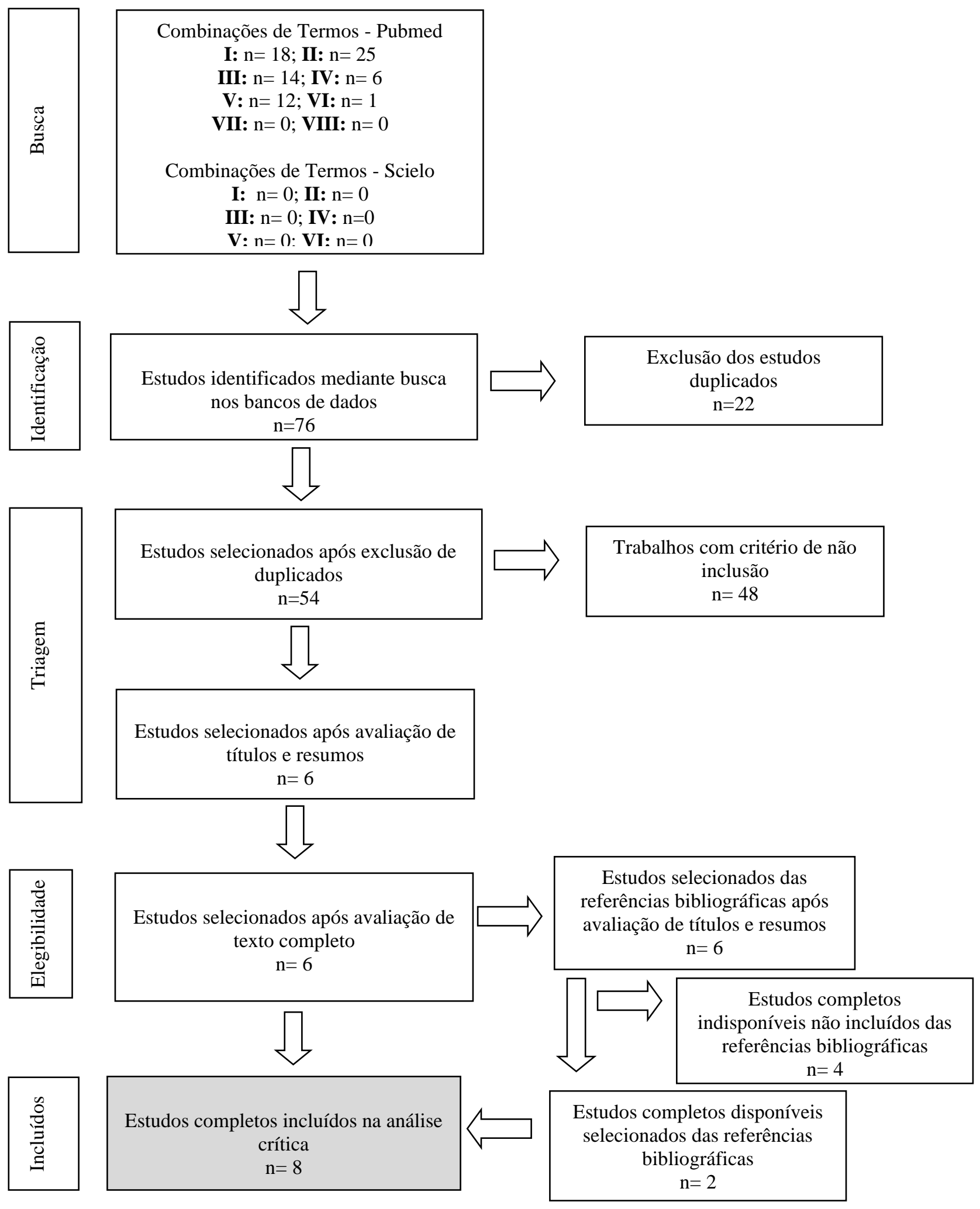

Legenda: $\mathrm{n}=$ número. Fonte: Autores (2021). 
Tabela 1. Descrição dos artigos selecionados.

\begin{tabular}{|c|c|c|c|c|c|}
\hline AUTOR/REF & $\begin{array}{l}\text { TIPO DE } \\
\text { ESTUDO, N } \\
\text { E PERÍODO }\end{array}$ & OBJETIVO & INTERVENÇÃO & RESULTADOS & CONCLUSÃO \\
\hline $\begin{array}{l}\text { Sampalis et al. } \\
(2003)\end{array}$ & $\begin{array}{l}\text { Duplo-cego, ensaio } \\
\text { clínico randomizado } \\
70 \text { mulheres. } \\
90 \text { dias. }\end{array}$ & $\begin{array}{l}\text { Avaliar a eficácia } \\
\text { do Neptune Krill } \\
\text { Oil тм (NKO тM) } \\
\text { para o manejo da } \\
\text { síndrome pré- } \\
\text { menstrual (SPM) } \\
\text { e DP. Comparar a } \\
\text { eficácia do NKO } \\
\text { com o óleo de } \\
\text { peixe. }\end{array}$ & $\begin{array}{l}\text { Administração oral: } \\
\text { GRUPO I: } 1 \mathrm{~g} \text { do óleo de Krill } \\
\text { (18\% de EPA e } 12 \% \text { de DHA) } \\
\text { GRUPO II: } 1 \mathrm{~g} \text { do óleo de } \\
\text { peixe (18\% de EPA e } 12 \% \text { de } \\
\text { DHA). }\end{array}$ & $\begin{array}{l}\text { GRUPO I: Após intervalos de } 45 \text { dias e } 90 \text { dias } \\
\text { redução significativa }(\mathrm{p}<0,001) \text { de sintomas } \\
\text { físicos e emocionais. } \\
\text { GRUPO II: Após } 90 \text { dias redução significativa } \\
\text { apenas de ganho de peso ( } \mathrm{p}<0,01) \text {, dor } \\
\text { abdominal ( } \mathrm{p}<0,001) \text { e edema }(\mathrm{p}<0,001) \text {. }\end{array}$ & $\begin{array}{l}\text { Sugerem que } 1 \mathrm{~g} \text { do óleo de Krill é } \\
\text { significativamente mais eficaz para } \\
\text { o controle da DP e sintomas } \\
\text { emocionais da SPM do que óleo de } \\
\text { peixe. }\end{array}$ \\
\hline $\begin{array}{l}\text { Moghadamnia } \\
\text { et al. (2010) }\end{array}$ & $\begin{array}{l}\text { Estudo cruzado de } 6 \\
\text { ciclos, duplo-cego, } \\
\text { randomizado, } \\
\text { controlado por } \\
\text { placebo. } \\
36 \text { mulheres. } \\
6 \text { meses ( } 3 \text { meses de } \\
\text { suplemen-tação). }\end{array}$ & $\begin{array}{l}\text { Investigar o papel } \\
\text { da suplementação } \\
\text { dietética com óleo } \\
\text { de } C \text {. Grimmi } \\
\text { (óleo de peixe) } \\
\text { sobre os sintomas } \\
\text { da DP em } \\
\text { meninas jovens. }\end{array}$ & $\begin{array}{l}\text { Administração oral: } \\
\text { GRUPO I: } 15 \mathrm{ml} \text { da emulsão do } \\
\text { óleo de peixe ( } 755 \text { mg de } \\
\text { ômega-3: } 550 \mathrm{mg} \text { de ácido EPA } \\
\text { e } 205 \mathrm{mg} \text { de DHA), durante os } \\
\text { primeiros } 3 \text { meses, seguido de } \\
\text { placebo por mais } 3 \text { meses. } \\
\text { GRUPO II: } 15 \mathrm{ml} \text { da emulsão } \\
\text { do placebo, iniciando com } \\
\text { placebo e mudança para o óleo } \\
\text { de peixe. }\end{array}$ & $\begin{array}{l}\text { Os grupos apresentaram uma diferença } \\
\text { significativa }(\mathrm{p}<0,001) \text { ao fim de } 3 \text { meses de } \\
\text { suplementação com o óleo de peixe comparado } \\
\text { ao placebo. Houve redução acentuada }(\mathrm{p}<0,005) \\
\text { na dor lombar e dor abdominal, e os participantes } \\
\text { utilizaram significativamente }(\mathrm{p}<0,001) \text { menos } \\
\text { doses de resgate de ibuprofeno durante a } \\
\text { utilização de óleo de peixe. }\end{array}$ & $\begin{array}{l}\text { Suplementação dietética com óleo } \\
\text { de } C \text {. Grimmi contendo ômega-3 foi } \\
\text { eficaz no alívio dos sintomas de DP. } \\
\text { O efeito marcante do ômega-3 } \\
\text { provavelmente resulta da produção } \\
\text { de PGs e LT antiinflamatórias. }\end{array}$ \\
\hline $\begin{array}{l}\text { Zafari et al. } \\
\text { (2011a) }\end{array}$ & $\begin{array}{l}\text { Ensaio clínico } \\
\text { randomizado, } \\
\text { duplo-cego. }\end{array}$ & $\begin{array}{l}\text { Comparar os } \\
\text { efeitos do óleo de } \\
\text { peixe e da } \\
\text { acupuntura com } \\
\text { as pílulas de }\end{array}$ & $\begin{array}{l}\text { Administração oral: } \\
\text { GRUPO I: dosagem diária de } \\
1 \mathrm{~g} \text { de óleo de peixe. Ele contém }\end{array}$ & $\begin{array}{l}\text { Quanto à intensidade da dor antes e após a } \\
\text { intervenção, houve diferença estatisticamente } \\
\text { significante no grupo I }(\mathrm{p}=0,000) \text {, o grupo II ( } \mathrm{p} \\
=0,000) \text { e o grupo III }(\mathrm{p}=0,000) \text {. Em relação à } \\
\text { duração da dor antes e após o tratamento, houve }\end{array}$ & $\begin{array}{l}\text { Cápsulas de óleo de peixe e de } \\
\text { acupuntura (têm poucos efeitos } \\
\text { colaterais e são muito aceitável e } \\
\text { tolerável) podem substituir }\end{array}$ \\
\hline
\end{tabular}


196 mulheres.

3 meses. ibuprofeno na DP

gordura saturada $(1 \mathrm{~g}), \mathrm{EPA}$

$(180 \mathrm{mg})$ e DHA $(120 \mathrm{mg})$

GRUPO II: ibuprofeno 400mg

GRUPO III: acupuntura

\begin{tabular}{|c|c|c|c|}
\hline $\begin{array}{l}\text { Zafari et al. } \\
\text { (2011b) }\end{array}$ & $\begin{array}{l}\text { Ensaio clínico } \\
\text { randomizado, } \\
\text { duplo-cego. } \\
120 \text { mulheres. } \\
2 \text { meses. }\end{array}$ & $\begin{array}{l}\text { Comparara } \\
\text { eficácia de óleo } \\
\text { de peixe e } \\
\text { ibuprofeno no } \\
\text { tratamento de } \\
\text { DP. }\end{array}$ & $\begin{array}{l}\text { Administração oral: } \\
\text { GRUPO I: dosagem } \\
\text { 1g de óleo de peixe } \\
\text { GRUPO II: ibuprofe } \\
\text { 400mg (quando a dor } \\
\text { começasse e se contin } \\
\text { tomariam a mesma do } \\
\text { horas). }\end{array}$ \\
\hline $\begin{array}{l}\text { Rahbar et al. } \\
\text { (2012) }\end{array}$ & $\begin{array}{l}\text { Estudo cruzado } \\
\text { duplo-cego. } \\
95 \text { mulheres. } \\
3 \text { meses }\end{array}$ & $\begin{array}{l}\text { Avaliar a eficácia } \\
\text { de ômega-3 no } \\
\text { tratamento de } \\
\text { mulheres com DP. }\end{array}$ & $\begin{array}{l}\text { Administração oral: } \\
\text { Inicialmente: }\end{array}$ \\
\hline
\end{tabular}

GRUPO I - ómega-3: 3

cápsulas de ômega $3(180 \mathrm{mg}$

EPA e 120 mg DHA).

\section{GRUPO II- placebo}

Após um washout de 1 semana sem qualquer medicação. $O$ grupo 1 , em seguida, receberan placebo durante 3 meses. $\mathrm{O}$ diferença significante no grupo II $(\mathrm{p}=0,000)$, o grupo I ( $p=0,000)$ e o grupo III $(p=0,000)$. A intensidade da dor após o tratamento não foi a mesma nos três grupos, apenas para os grupos II III $(\mathrm{p}=0,000)$. A duração da dor após o tratamento também não foi a mesma para os três grupos $(\mathrm{p}=0,000)$. Os três grupos tiveram a mesma necessidade de analgésicos extras $(\mathrm{p}=$ 0,295 ), mas houve uma diferença significativa no grau de satisfação nos três grupos $(\mathrm{p}=0,000)$ : $\mathrm{O}$ grupo que tomou ibuprofeno foi o mais satisfeito, seguido pelo acupuntura e os grupos de óleo de peixe.

\section{Houve uma diferença significativa entre a} intensidade da dor antes e depois do tratamento com ibuprofeno $(\mathrm{p}=0,001)$ e óleo de peixe $(\mathrm{p}=$ $0,001)$. Além disso, a reducão da dor no grupo de óleo de peixe foi maior que o grupo do Ibuprofeno $(p=0,001)$ fármacos AINE (que têm muitos efeitos secundários) na cura de pessoas com DP. Recomenda-se também que diferentes níveis de óleo de peixe devem ser utilizados em estudos futuros.

\section{Os resultados mostram que a}

eficácia do óleo de peixe é melhor

do que o ibuprofeno no tratamento da dor severa na DP.

\section{A média da intensidade da dor foi}

significativamente reduzida entre as mulheres que receberam o ômega-3 em comparado ao placebo. A diferença entre os 2 grupos, neste tempo de 3 meses foi estatisticamente significativa $(\mathrm{p}=$ $0,001)$.

Depois do cruzamento, a pontuação da

intensidade da dor foi consistentemente mais baixa quando as mulheres receberam ômega- 3 do que quando eles receberam placebo $(\mathrm{P}<0,001)$. Além de diminuir a pontuação da intensidade da dor, tratamento com ômega-3 também reduziu a dose de resgate de ibuprofeno. A diferença entre os 2 grupos foi estatisticamente significativa em ambos os pontos de tempo $(\mathrm{P}=0,001)$
Indicam que a suplementação

dietética com ômega-3 pode levar à redução da dor perceptível entre as mulheres jovens com DP. Além disso, a utilização de ômega-3 reduziu a dose resgate de ibuprofeno necessária para controlar a dor menstrual extrema. Uma limitação potencial do presente estudo foi o período de washout curto (1 semana). No entanto, o uso de ômega-3 poderia oferecer uma alternativa aos AIEN para o tratamento seguro e eficaz na DP. 
meses.

\begin{tabular}{|c|c|c|c|c|c|}
\hline $\begin{array}{l}\text { Hosseinlou et } \\
\text { al. (2014) }\end{array}$ & $\begin{array}{l}\text { Ensaio clínico } \\
\text { duplo-cego } \\
\text { randomizado } \\
\text { controlado por } \\
\text { placebo. } \\
240 \text { mulheres. } \\
2 \text { meses. }\end{array}$ & $\begin{array}{l}\text { Determinar o } \\
\text { efeito da vitamina } \\
\text { B1 (VIT B1) e } \\
\text { óleo de peixe em } \\
\text { conjunto na } \\
\text { gravidade e } \\
\text { duração da DP. }\end{array}$ & $\begin{array}{l}\text { Administração oral: } \\
\text { GRUPO I: Vitamina B1 (100 } \\
\text { mg/dia) } \\
\text { GRUPO II: Ômega3 (óleo de } \\
\text { peixe de } 500 \text { mg/dia). } \\
\text { GRUPO III:Vitamina B1 + } \\
\text { Ômega } 3\end{array}$ & $\begin{array}{l}\text { Em todas as drogas, os resultados (média) foi } \\
\text { melhor no final do segundo mês do que no } \\
\text { primeiro mês. A intensidade de dor durante dois } \\
\text { meses após a intervenção mostrou uma } \\
\text { diminuição estatisticamente significativa entre os } \\
\text { três grupos em comparação com o grupo de } \\
\text { placebo (p <0,001). A duração da dor durante } \\
\text { dois meses após a intervenção mostrou uma } \\
\text { diminuição significativa nos três grupos, em } \\
\text { comparação com o grupo placebo }(\mathrm{P}<0,05) \text {. }\end{array}$ & $\begin{array}{l}\text { Efeitos positivos da VIT B1 e óleo } \\
\text { de peixe sobre os sintomas da DP e } \\
\text { sugerem substituir os fármacos } \\
\text { AINE (que têm muitos efeitos } \\
\text { secundários). Recomendam novas } \\
\text { pesquisas, utilizando maior } \\
\text { quantidade de doses de óleo de } \\
\text { peixe e VIT B1 em toda a duração } \\
\text { do ciclo, mais tempo e períodos de } \\
\text { tratamento mais longos. }\end{array}$ \\
\hline & & & GRUPO IV: placebo & & \\
\hline $\begin{array}{l}\text { Mehrpooya et } \\
\text { al. }(2017)\end{array}$ & $\begin{array}{l}\text { Ensaio clínico } \\
\text { duplo- cego } \\
\text { randomizado. } \\
80 \text { mulheres. } \\
3 \text { meses. }\end{array}$ & $\begin{array}{l}\text { Comparar a } \\
\text { eficácia de óleo } \\
\text { de peixe com o } \\
\text { cálcio na DP. }\end{array}$ & $\begin{array}{l}\text { Administração oral: } \\
\text { GRUPO I: Õmega } 3(1 \mathrm{~g})+ \\
\text { Ibuprofeno ( } 400 \mathrm{mg} \text { - caso } \\
\text { necessário) } \\
\text { GRUPO II: Cálcio }(1 \mathrm{~g})+ \\
\text { Ibuprofeno ( } 400 \mathrm{mg} \text { - caso } \\
\text { necessário) } \\
\text { As drogas e suplementos foram } \\
\text { utilizados todos os dias no } \\
\text { primeiro ciclo e a partir de } 8 \\
\text { dias antes até } 2 \text { dias após o } \\
\text { início da menstruação, para os } \\
\text { segundo e terceiro ciclos. }\end{array}$ & $\begin{array}{l}\text { Houve diferença estatisticamente significante } \\
\text { entre o grupo I e II em relação a náuseas, } \\
\text { vômitos, sensibilidade mamária, acne, cefaléia e } \\
\text { inchaço após um mês, mas não houve diferença } \\
\text { estatisticamente significante entre o grupo I e II } \\
\text { em relação a acne e dor de cabeça após } 2 \text { meses e } \\
3 \text { meses (p>0,05). Não houve diferença } \\
\text { significativa para intensidade de dor entre os } \\
\text { grupos I e II antes da intervenção e após o final } \\
\text { do primeiro ciclo (p>0,05). No entanto, houve } \\
\text { uma diferença significativa entre os grupos I e II } \\
\text { no final do segundo mês (P = 0,001) e o terceiro } \\
\text { mês (p <0,001). Houve uma diferença } \\
\text { significativa nos escores de intensidade da dor } \\
\text { menstrual no primeiro, segundo e terceiro ciclo } \\
\text { menstrual, em comparação com os escores antes } \\
\text { da intervenção (p<0,001) para o grupo I. } \\
\text { Houve diferença estatisticamente significante } \\
\text { entre média + desvio padrão do consumo de }\end{array}$ & $\begin{array}{l}\text { Suplementação de ômega-3 e cálcio } \\
\text { podem ser eficazes no tratamento de } \\
\text { mulheres que sofrem de DP. No } \\
\text { entanto, concluíram que o ômega-3 } \\
\text { é mais eficaz do que o cálcio }\end{array}$ \\
\hline
\end{tabular}


redução foi mais no grupo I do que grupo II.

\begin{tabular}{|c|c|c|c|c|c|}
\hline $\begin{array}{l}\text { Sadeghi et al. } \\
\text { (2018) }\end{array}$ & $\begin{array}{l}\text { Ensaio clínico } \\
\text { duplo-cego } \\
\text { randomizado } \\
\text { controlado por } \\
\text { placebo. } \\
100 \text { mulheres } \\
2 \text { meses. }\end{array}$ & $\begin{array}{l}\text { Investigar o efeito } \\
\text { adjuvante da } \\
\text { vitamina E (VIT } \\
\text { E) ômega-3, } \\
\text { separadamente ou } \\
\text { em combinação, } \\
\text { sobre dor no } \\
\text { tratamento da DP. }\end{array}$ & $\begin{array}{l}\text { Administração oral: } \\
\text { GRUPO I -ômega-3 (n 1/4 } \\
\text { 25):dosagem diária de } 300 \mathrm{mg} \\
\text { de ômega } 3 \text { (180 mg EPA e } 120 \\
\text { mg DHA). } \\
\text { GRUPO II- VIT E (n 1/4 25):A } \\
\text { dose diária de VIT E (acetato de } \\
\text { alfa-tocoferol) foi de } 200 \\
\text { unidades internacionais (UI). } \\
\text { GRUPO III VIT E- ômega-3 } \\
\text { (n 1/4 25): } 300 \text { g ômega } 3+ \\
\text { 200UI VIT E } \\
\text { GRUPO IV- ou placebo (n 1/4 } \\
\text { 25). } \\
\text { Cada participante tomou as } \\
\text { cápsulas por } 5 \text { dias ( } 2 \text { dias antes } \\
\text { e } 3 \text { dias após o início da } \\
\text { menstruação). }\end{array}$ & $\begin{array}{l}\text { Redução da dor menstrual foi relatada nos grupos } \\
\text { de intervenção. O Grupo III tem um efeito } \\
\text { considerável sobre a dor menstrual, quando } \\
\text { comparados com outros grupos }(\mathrm{p}<0,05) \text {. }\end{array}$ & $\begin{array}{l}\text { Ômega-3 e vitamina E reduziram } \\
\text { gravidade da dor da DP em dois } \\
\text { meses, mas que o ômega } 3 \text { em } \\
\text { combinação com a vitamina E teve } \\
\text { o maior efeito. }\end{array}$ \\
\hline
\end{tabular}

Fonte: Autores (2021). 
Esses autores, assim como Rahbar et al. (2012) sugerem que a utilização dos AGE facilita a redução da síntese de PG e LT pro-inflamatórios, além de aumentar a produção de PG benéficas antiespasmódicas e anti-inflamatórias com mecanismo de ação similar a determinadas drogas anti-inflamatórias. Esse mecanismo de ação ocorre pelo aumento da ingestão de ômega-3, em particular ácido eicosapentaenóico (EPA), que irá inverter os sintomas da DP pela inibição competitiva no sítio de ação da ciclo-oxigenase. Assim, resulta-se em menor formação de derivados de ácido araquidônico (AA), como PGF2- $\alpha$, PGE2 e LTB4 e aumenta-se a formação derivados do EPA, como a PGE3 (Andrade \& Carmo, 2006) (Martin et al.,2006), por exemplo, ocasionando efeitos positivos do ômega 3 na redução de sintomas de DP.

Em relação ao tempo de uso da suplementação do ômega 3 para observar o seu efeito, verificou-se no estudo de Rahbar et al. (2012) que após 3 meses, a média da intensidade da dor foi significativamente reduzida entre as mulheres que receberam o ômega-3 em relação ao placebo. Assim como no estudo de Moghadamnia et al. (2010) revelou uma redução estatisticamente significativa após 3 meses de suplementação com óleo de peixe em comparação com placebo, apresentando uma redução significativa na dor abdominal e lombar no grupo que recebeu o óleo de peixe no final dos meses 2 e 3 do estudo. Sampalis et al. (2003) demonstraram que o óleo de Krill após intervalos de 45 dias (primeiro ciclo menstrual) e 90 dias (segundo e terceiro ciclo) reduziram significativamente em ambos os sintomas emocionais e físicos relacionados SPM e DP. Zafari et al. (2011a) observaram que a intensidade e a duração da dor diminuíram no grupo suplementado por óleo de peixe a final de 3 meses. Mehrpooya et al. (2017) avaliaram sinais clínicos a cada ciclo menstrual em 3 meses e encontraram resultados mais promissores entre 2 e 3 meses de intervenção. Zafari et al. (2011b), Hosseinlou et al. (2014) e Sadeghi et al. (2018) obtiveram resultados positivos após 2 meses de intervenção.

Nenhum artigo traz explicação quanto o tempo de uso, contudo verifica-se que a partir de 2 meses de uso já é possível observar efeitos. No entanto, chama-se atenção para o tempo de uso de acordo com os sinais clínicos apresentados. Há variação entre os estudos quanto a forma de uso e as doses de suplementação de ômega 3 recomendada para obter efeitos. A dose menor e mais utilizada entre os estudos foi de 300mg (Sampalis, 2003) (Zafari et al., 2011a) (Rahbar et al., 2012) (Sadaghi et al., 2018) com resultados significativamente positivos. No protocolo de intervenção de Mehrpooya et al. (2017) o suplemento foi administrado todos os dias no primeiro ciclo e de 8 dias antes até 2 dias após o início da menstruação para o segundo e terceiro ciclo. Já Sadeghi et al. (2018) administraram durante cinco dias (dois dias antes e três dias depois do início menstruação) em cada ciclo. Observa-se a necessidade de protocolos mais definidos quanto a forma de uso e as doses da suplementação de ômega 3, no entanto, haja vista os efeitos positivos e ausência de efeitos colaterais, cabe sugerir o uso conforme os protocolos encontrados nos ensaios clínicos estudados.

Sampalis et al. (2003) discutem o efeito dos diferentes perfis de ômega 3 ofertados no óleo de Krill e peixe para melhora dos sinais clínicos em mulheres com DP. Em peixes, os ácidos graxos são armazenados principalmente como triglicerídeos, enquanto no krill, 30-65\% dos ácidos graxos são incorporados em fosfolipídios (Sampalis, 2003) (Ulven et al., 2011). Os autores sugerem que $1 \mathrm{~g}$ do óleo de Krill é significativamente mais eficaz para o controle da DP e sintomas emocionais da síndrome pré-menstrual (SPM) do que óleo de peixe. Isso ocorre devido o perfil de ômega-3 do óleo de Krill facilitar a passagem de moléculas de ácidos graxos através da parede intestinal, aumentando a sua biodisponibilidade, melhorando a proporção ômega-3/ômega-6 e potenciais efeitos de modulação sobre neurotransmissores que afetam os sintomas emocionais e psicológicos. Além disso, os potenciais efeitos benéficos associados ao óleo de krill podem ser também atribuídos ao conteúdo de antioxidantes como as vitaminas A e E, além da astaxantina (Sampalis, 2003). De modo que os autores concluem que o suplemento de óleo de krill é seguro, bem tolerado e representa uma fonte valiosa de ômega 3 no tratamento de SPM e DP (Sampalis, 2003). 
Além dos estudos que investigam o uso isolado de omêga 3 para o tratamento da DP, alguns ensaios clínicos (Mehrpooya et al., 2017) (Hosseinlou, et al., 2014) (Sadaghi et al., 2018) avaliam a suplementação de nutrientes combinados ao ômega 3 para conseguir efeitos sinérgicos na terapêutica da DP. No estudo de Sadeghi et al. (2018) tanto ômega-3 quanto a vitamina E reduziram a gravidade da dor da DP em dois meses, mas o ômega 3, em combinação com a vitamina E, teve o maior efeito. Os resultados foram semelhantes no estudo de Ziaei, Zakeri e Kazemnejad (2005) que demonstram que a vitamina E reduziu a gravidade e a duração da dor de DP e reduziu a quantidade de perda de sangue menstrual. Todos estes efeitos podem ser atribuídos à redução da síntese de PG por vitamina $\mathrm{E}$ que atua como antioxidante. A vitamina $\mathrm{E}$ reduz a síntese de PG, por ser inibidor da proteína quinase C, evitando peroxidação dos fosfolipídica e liberação de ácido araquidônico a partir de membranas celulares) (Sadaghi et al., 2018). Dessa forma, a vitamina E pode potencializar os efeitos do ômega 3 com sua função antioxidante.

Outro nutriente investigado em comparação ao ômega 3 foi a vitamina B1. A vitamina B1 é utilizada no corpo para atividades do sistema muscular e nervoso e pode afetar de forma positiva sobre a contração muscular do útero (Hosseinlou, et al., 2014). No estudo de Hosseinlou et al. (2014) comparou-se o efeito terapêutico de vitamina B1, óleo de peixe e terapia de combinação no alívio da intensidade e duração da dor. Nesse estudo, encontrou-se resultados significativamente positivos em relação ao placebo e sem diferenças significativas entre os grupos experimentais suplementados. Um outro estudo que compara apenas uso de vitamina B1 em relação ao ibuprofeno discute que os dois produzem efeitos semelhantes, mas vitamina B1 tem menos complicações sendo mais aceita no tratamento da DP (Zafari, Aghamohammady \& Tofighi, 2011). O uso da vitamina B1 e ômega 3 tem resultados semelhantes embora tenham mecanismos de ação diferentes, porém não se observou efeito sinérgico com a terapia de combinação no ensaio clinico analisado (Hosseinlou, et al., 2014).

Os efeitos da suplementação de cálcio, também, são estudados em comparação aos do ômega 3, já que a desregulação de cálcio em resposta à mudança hormonal durante o ciclo menstrual pode influenciar a ocorrência de dor (Mehrpooya et al., 2017). O estrógeno atua na regulação do metabolismo do cálcio, na absorção intestinal e na secreção deste mineral (Sampaio, 2002). A queda deste hormônio durante a menstruação leva a diminuição da disponibilidade do cálcio e consumo da suplementação deste pode ter algum benefício em mulheres com SPM e DP (Ghanbari, Haghollahi, Shariat, Foroshani \& Ashrafi, 2009). O ensaio clínico de Mehrpooya et al. (2017) evidenciou que a suplementação de ômega-3 e cálcio podem ser eficazes no tratamento de mulheres que sofrem de DP. No entanto, concluíram que o ômega-3 é mais eficaz do que o cálcio.

Por fim, o único fator limitante ao uso de ômega 3 foi descrito no estudo de Moghadamnia et al. (2010) atribuindo o sabor desagradável do óleo de peixe como um fator de perda de continuidade do tratamento.

\section{Conclusão}

Nesta pesquisa foi observado que o ômega 3 foi eficaz e sua ação ocorre pela inibição da atividade da ciclo-oxigenase, que leva a menor produção de PG e LT pró-inflamatórias, aumentando a formação de seus derivados, anti-inflamatórios. A posologia recomendada com mais frequência é a de $300 \mathrm{mg}$ de ômega-3, 8-2 dias antes até 3-2 dias depois da menstruação a cada ciclo, capaz de reduz dor perceptível entre as mulheres jovens com DP, principalmente após 2 meses de uso.

Além disso, verificou-se que a combinação do ômega 3 com a vitamina $E$, devido este último ser um potente antioxidante, pode potencializar a redução de prostaglandinas. Identificou-se ainda que o ômega 3 pode ter resultados semelhantes a vitamina B1 e superior a suplementação de cálcio. Ademais, o óleo de Krill é uma alternativa segura e bem tolerada e representa uma fonte valiosa de ômega 3, além de ser mais eficaz no controle de sintomas emocionais.

Esta revisão apresenta algumas limitações devido o número pequeno de estudos que avaliam suplementação com AGE no tratamento da DP. Além disso, observa-se baixa produção de ensaios clínicos disponíveis que avaliem exclusivamente 
o AGE ômega 6 (GLA) no tratamento da DP, demonstrando a existência de uma lacuna de conhecimento nesta abordagem clínica. No entanto, o presente estudo demonstra que os AGE podem oferecer uma alternativa ao uso de medicamentos frequentemente utilizado, como AINES no tratamento da DP. Assim, auxiliam na redução dos sintomas e limitam o impacto dessa condição nas atividades de vida diária e qualidade de vida dessas mulheres.

Neste contexto, torna-se evidente que há benefícios do uso de AGE na terapêutica suplementar da dismenorreia primária, a partir dos ensaios clínicos consultados. Entretanto, sugere-se ampliação das pesquisas, com maior número de indivíduos e, que avaliem a suplementação com AGE, como o uso do AGE ômega 6 (GLA) no tratamento da DP, possibilitando trabalhos futuros acerca dessa temática.

\section{Referências}

Alves, T. P., Yamagishi, J. A., da Silva N. J., Júnior A. T. T \& Lima R. R. O. (2016). Dismenorreia: Dianóstico e Tratamento. Revista Científica da Faculdade de Educação e Meio Ambiente, 7(2): 1-12.

Andrade, P. D. M. M. \& Carmo, M. G. T. (2006). Ácidos graxos n-3: um link entre eicosanóides, inflamação e imunidade. Revista de Metabolismo e Nutrição, 8(3): 135-143.

Araújo L. M., da Silva, J. M. N, Bastos, W. T. \& Ventura, P. L. (2012). Diminuição da dor em mulheres com dismenorreia primária, tratadas pelo método Pilates. Revista Dor, 13 .

Dall'Acqua, R. \& Bendlin, T. (2015). Dismenorreia. Femina, 43(6).

Dardes, R. D. C., Moraes, A.S. \& Santos, M. B. (2011) Como diagnosticar e tratar dismenorreia. RBM, 68(12): 14-26.

Estrela, C. (2018). Metodologia Científica: Ciência, Ensino, Pesquisa. Editora Artes Médicas.

Gerzson, L. R., Padilha, J. F., Braz, M. M. \& Gasparetto, A. (2014). Physiotherapy in primary dysmenorrhea: literature review. Revista Dor, 15(4): 290-295.

Ghanbari, Z., Haghollahi, F., Shariat, \& Foroshani, A. R, Ashrafi M. (2009). Effects of calcium supplement therapy in women with premenstrual syndrome. Taiwanese Journal of Obstetrics and Gynecology, 48(2): 124-129.

Giraldo, P. C., Eleutério Júnior, J. \& Linhares IM. (2008). Dismenorréia. RevBras Med, 65(6): 164-168.

Hosseinlou, A., Alinejad, V., Alinejad, M. \& Aghakhani, N. (2014). Effects of Fish Oil Capsules and Vitamin B1 Tablets on Duration and Severity of Dysmenorrhea in Students of High School in Urmia-Iran. Global Journal of Health Science, 6(7): 124.

Hudson, T. (2007). Using nutrition to relieve primary dysmenorrhea. Alternative \& Complementary Therapies, 13(3):125-128.

Koche, J. C. (2011). Fundamentos de metodologia científica. Petrópolis: Vozes. http://www.brunovivas.com/wpcontent/uploads/sites/10/2018/07/K\%C3\%B6che-Jos\%C3\%A9-Carlos0D0AFundamentos-de-metodologia-cient\%C3\%ADfica-_-teoria-da0D0Aci\% C3\%AAncia-e-inicia\%C3\%A7\%C3\%A3o-\%C3\%A0-pesquisa.pdfhttps://repositorio.ufsm.br/bitstream/handle/1/15824/Lic_Computacao_MetodologiaPesquisa-Cientifica.pdf?sequence $=1$

Ludke, M. \& Andre, M. E. D. A. (2013). Pesquisas em educação: uma abordagem qualitativa. São Paulo: E.P.U. 5.5) Yin, R.K. (2015). O estudo de caso. Porto Alegre: Bookman.

Martin, C. A., de Almeida, V. V., Ruiz, M. R., Visentainer, J. E. L., Matshushita, M., de Souza, N. E. \& Visentainer, J. V. (2006) Ácidos graxos poliinsaturados ômega-3 e ômega-6: importância e ocorrência em alimentos Ômega-3 and ômega-6 polyunsaturatedfattyacids: importanceandoccurrence in foods. Revista de Nutrição, 19(6): 761-770.

Mayo, J. L. (1997). A healthy menstrual cycle. Clin Nutr Insights, 5(9): 1-8.

Mehrpooya, M., Eshraghi, A., Rabie, S., Larki-Harchegani, A. \& Ataei, S. (2017). Comparison the Effect of Fish-Oil and Calcium Supplementation on Treatment of Primary Dysmenorrhea. Reviews on recent clinical trials, 12(3):148-153.

Moghadamnia, A. A., Mirhosseini, N., Abadi M. H., Omranirad, A., Omidvar, S. (2010). Effect of Clupeonellagrimmi (anchovy/kilka) fish oil on dysmenorrhoea. Eastern Mediterranean Health Journal, 16(4).

Pereira A. S. et al. (2018). Metodologia da pesquisa científica. [free e-book]. Santa Maria/RS. Ed. UAB/NTE/UFSM.

Rahbar, N., Asgharzadeh, N. \& Ghorbani, R. (2012) Effect of ômega-3 fatty acids on intensity of primary dysmenorrhea. International Journal of Gynecology \& Obstetrics, 117(1): 45-47.

Sadeghi, N., Paknezhad, F., Rashidi, N. M., Kavianpour, M., Jafari, R. S. \& Khadem, H. H. (2018). Vitamin E and fish oil, separately or in combination, on treatment of primary dysmenorrhea: a double-blind, randomized clinical trial. Gynecological Endocrinology, 1-5.

Sampaio, H. A. D. C. (2002). Aspectos nutricionais relacionados ao ciclo menstrual. Revista de Nutrição, 15(3):309-317. 
Research, Society and Development, v. 10, n. 14, e191101421732, 2021

(CC BY 4.0) | ISSN 2525-3409 | DOI: http://dx.doi.org/10.33448/rsd-v10i14.21732

Sampalis, F., Bunea, R., Pelland, M. F., Kowalski, O., Duguet, N. \& Dupuis, S. (2003). Evaluation of the effects of Neptune Krill Oil ${ }^{\mathrm{TM}}$ on the management of premenstrual syndrome and dysmenorrhea. Alternative Medicine Review, 8(2): 171-179.

Sezeremeta, D. C., dos Santos, C. M. S, Vrecchi, M. R., Marafon, R. G. C., Crespilho, L. C., Pagotto, J. P. \& Mortean, E. D. C. M. (2015). Dismenorreia: Ocorrência na vida de acadêmicas da área de saúde. Journal of Health Sciences, 15(2).

Ulven, S. M., Kirkhus, B., Lamglait, A., Basu, S., Elind, E. \& Haider, T., Pedersen, J. I. (2011). Metabolic effects of krill oil are essentially similar to those of fish oil but at lower dose of EPA and DHA, in healthy volunteers. Lipids, 46(1): 37-46.

Zafari, M., Tofighi, M., Aghamohammady, A., Behmanesh, F. \& Rakhshaee, Z. (2011a). Comparison of the effect of acupressure, fish oil capsules and ibuprofen on treatment of primary dysmenorrhea. Afr J Pharm Pharmacol, 5(8): 1115-9.

Zafari, M., Behmanesh, F. \& Mohammadi, A. A. (2011b). Comparison of the effect of fish oil and ibuprofen on treatment of severe pain in primary dysmenorrhea. Caspian journal of internal medicine, 2(3): 279.

Zafari, M., Aghamohammady, A. \& Tofighi M. (2011) Comparing the effect of vitamin B1 (vit. B1) and ibuberofen on the treatment of primary dysmenorrhea. Afr. J. Pharm. Pharmacol, 5(7): 874-878.

Ziaei, S., Zakeri, M., Kazemnejad, A. (2005). A randomised controlled trial of vitamin E in the treatment of primary dysmenorrhoea. BJOG, $112(8): 1164$. 